BULL. AUSTRAL. MATH. SOC.

MOS $6210,6297,6220$

VOL. $2(1970), 137$.

\title{
Mixtures of distributions
}

\section{J. Robinson}

The thesis was in four not closely related parts, each of which, however, involved mixtures of distributions.

In the first part, a theorem giving necessary and sufficient conditions for the identifiability of a class of distributions, was obtained by applying Choquet's uniqueness theorem on representations of convex sets as means of the set of their extreme points. The theorem was used to prove the identifiability of three classes of mixtures.

Part Two was a study of mixtures of normal and multivariate normal distributions.

In the third part mixture representations were obtained for the distributions of definite and non-definite quadratic forms of normal variates and for ratios of quadratic forms.

The fourth part contained a method of estimating the carrier rate from amoebic surveys. This involved estimating parameters from a mixture of geometric distributions. The methods were applied to some sets of published data and a simulation experiment was performed to determine the order of errors inherent in the estimation procedure.

Received 25 March 1969. Thesis submitted to the University of Sydney, 13 August 1968. Degree approved, 28 February 1969. Supervisor: Professor H.o. Lancaster. 